\title{
31 ON THE ASSEMBLY LINE: A VIEW FROM INDUSTRIAL SOCIOLOGY
}

\author{
Paula Urze ${ }^{1}$, Tiago Machado ${ }^{2}$ \\ ${ }^{\prime}$ FCT/UNL - Faculdade de Ciências e Tecnologia, Universidade Nova de Lisboa \\ ${ }^{2}$ UNINOVA - Instituto de Desenvolvimento de Novas Tecnologias \\ pcu@fct.unl.pt; tgm@uninova.pt
}

\begin{abstract}
This paper presents the scientific concerns and some restults of the LiMITE - on the Assembly Line: Innovation, Work and Employment-project. Even considering the vast number of experimented industry production technologies, the classic assembly line has proved to be a structuring element within the frame of mass production, whereas known the difficulty to find practical alternative solutions. In this sense, the constraints brought up by the assembly line in order to develop innovative working practices remain relevant. For this reason, it is also important to look for the possibility to develop innovative working practices under the dominant model, i.e. the assembly line inherited from Ford. The paper's empirical section is based on two case studies pointed to two companies from the automotive components industry.
\end{abstract}

\section{INTRODUCTION}

Even thought the design of production systems has always been considered a crucial problem to industrial engineers, the global competition and the fast evolution of production technologies have contributed to increase its importance. Rekiek et al. (2002) point out that production systems are nowadays characterized by shorter cycle times, higher automation levels and by the emergence of new production equipments.

Naturally, the configuration of assembly lines has been evolving since Henry Ford's era. Besides the traditional line, based on an unidirectional sequential workflow, and designed to assemble just one model, there is nowadays a multiplicity of new and more flexible configurations like mixed-model and multi-model lines, asynchronous lines interleaved by buffers, U-shaped lines, among other variants.

In 1973, the European Economic Community announced that the assembly line would have to be abolished from the European car industry (cf. Emery, 1975: 4). But after more than three decades, and considering the vast number of production technologies available today, the classic assembly line still seems to be an attractive industrial device for mass production. That is, even considering the vast number of experimented industry production technologies, the classic assembly line has proved to be a structuring element within the frame of mass production, whereas known the difficulty to find practical alternative solutions. In this sense, the constraints brought up by the assembly line in order to develop innovative working practices remain relevant.

For this reason, we shall keep on researching for the possibility to develop innovative working practices - surpassing assembly line repetitive work and the degradation of human labor - under the dominant model, i.e. the assembly line 
inherited from Ford. Are we going to experiment a return back to the old conflicts related to the repetitive work and to the work degradation or are there alternatives able to guarantee sustainability and attractiveness of the work on line?, asks Jürgens (1997). So we add a couple more questions: can mass production effectively discard the assembly line without lost of competitiveness? To what extent it continues to limit the existing options considering work organisation and its contents? Bringing to nowadays a classical expression (Trist et al., 1963) is there room for a genuine "organisational choice"?

\section{ORGANISATIONAL CHOICE (S)}

In a simplified assertion, the assembly line can be seen as a set of workplaces distributed sequentially and interconnected through a material transportation system. For each workplace a set of tasks are executed following a pre-defined assembly process which involves: (1) the time needed to execute the task; (2) the precedence relation between tasks establishing the execution sequence; (3) association zone restrictions in order to avoid the assignment of different tasks to the same workplace.

Assembly lines are synchronized in order to regulate the flow of components. Each workplace has a pre-defined time period to accomplish all associated tasks (cycle time). The transportation system is designed in such a way that when the cycle time is finished the subassembly that has been done in a workplace is transferred for the next workplace resulting in a new subassembly. When the time variability for tasks execution is high asynchronous lines can be used. In these lines, each workplace incorporates the assigned tasks at a certain rate. In this case the component is transferred to the next station only when all associated tasks are concluded. The decisions about the tasks to assign to each workplace in order to minimize the costs, satisfying market demand and simultaneously coping with precedence and zone restrictions, are the main challenges when designing an assembly line (assembly line balancing).

With more or less a century of existence, and although the recognition of some problems, the classic assembly line is still largely adopted in the worldwide industry. Actually, Jürgens reminds us (1997: 255-256) that the assembly line is since its inception a controversial socio-technique innovation. Before the World War II, the opinions about the effects of this technological instrument on the work quality and the workers satisfaction were divided. Later, in the $50 \mathrm{~s}$ and $60 \mathrm{~s}$, other voices criticizing the assembly line raised. In 1952, Walker e Guest, in The Man on the Assembly Line, wrote that "Of all occupations in modern industry none has attracted such controversial comment as that of the assembly line worker, and especially of the auto assembly worker on the 'final line'." The authors point out the negative effects of the assembly line for the automotive industry workers - mainly, the repetitive nature of the tasks and the "tight" dependency on the assembly line pace.

In 1964, Blauner, in Alienation and Freedom, emphasized the importance of the technological structure, namely of the assembly line, on the nature and organisation of work, in the following terms: "The assembly line's inexorable control over the pace and rhythm of work is most critical; it is largely responsible for the high degree of pressure, the inability to control the quantity of work, and the lack of free movement." Two years after, Goldthorpe concluded that "Assembly line production 
in the automobile industry is now generally regarded as the locus classicus of worker alienation." (Cf. Jürgens, 1997). Later, in the 70s, Coriat, in L'Atelier et le Chronometre, argued in the same direction: "A l'origine de la chaine, violence calculée, systématiquement appliquée contre le travail des hommes, ce rêve originel du capital à la recherche du 'mouvement perpétuel' de la fabrique. La production à flux continu, 'clé de voûte' de tous les systèmes d'organisation de travail (...)." (Coriat, 1994: 67).

We emphasise the Coriat approach due to the fact that, as Berggren (1992) underlines, ever since the edition of Organizational Choice in the 60s (Trist et al., 1963), a group of social scientists relied on the thesis that work organisation could be modeled almost independently from technological constraints - there would always be room for an "organizational choice". It is remarkable, adds the Swedish author that in the Trist et al. (1963) there's no support for this thesis, a perspective that can only be supported by a short term diagnostics but harder to sustain within the framework of longer studies, covering periods of economic expansion and contraction, plain employment and unemployment phases. Berggren's case studies clearly reveal the mutual interaction and interdependency between technical production design and work organisation.

Shimokawa et al. (1997) studied the alternatives to the traditional mass production. The authors begin to explain that the options related to the assembly systems adopted by the enterprises are influenced by their strategies and options and also by factors linked to the context and environment. Considering that the enterprises or regions deal with different objectives and constraints, assembly systems change. Within this framework, different assembly systems have been identified in the automobile industry: neo-fordist, Uddevalla, neo-craft and lean system. Revising the different models, the authors concluded that none of the existent alternative models has shown sustainable advantages comparing to traditional mass production system.

In Bergreen (1992), the alternatives to the traditional production system have been analysed. But in a more focused way, considering the configuration of the assembly systems as central dimension of analysis side by side with the work organisation dimension. It is important to underline the methodology developed by this work, due to the fact that, by articulating the technical and organizational dimensions, it emphasized the conditioning role of the technical subsystem in relation to the social system. This is, of course, the privileged focus of our research project.

At the end of this decade, Engström, Jonsson e Medbo (1998) tried to demonstrate the superiority (efficiency) of the assembly systems supported by an "organic" flow over the conventional assembly systems. Taking as a starting point the typology developed by Wild (1975), three type of losses were identified: (1) it is not possible to divide uniformly the work among the different workstations - losses associated to the balancing; (2) a large amount of work in the workstation is required for materials handling; (3) the assembler can not change his natural work rhythm loss associated to the system. Based on empirical information provided by the Swedish automobile industry, the authors conclude that the assembly system supported by an "organic" flow was more efficient than the conventional one. In fact, the sociological research related to alternatives to the assembly line leads mainly to the solutions experimented by Volvo. First in Kalmar's plant, and latter in 
the Udevalla plant. The interest in Volvo-ism has been intensified by the fact that the Uddevalla's unit, more innovative than its precedent, was closed in 1993 to reopen two years later under the name of Autonova (a result from the collaboration between Tom Wilkinshaw Racing and Volvo).

Udevalla is, among all the known cases (Toyota Kyûshû, Honda Flexifactiories, Volvo Kalmar, Uddevalla e Autonova, etc. ), the most emblematic one. Because it is an example of the "reflexive production" paradigm -42 parallel product workshop assembly (instead of a sequential assembly line), 480 minutes cycle time, working teams assembling the whole car and being responsible for planning, quality control, development of methods, etc. Uddevalla had exceptional conditions to promote socio-organisational innovation - "This plant was in all its aspects a humanistic production system." (Granath, 1998).

As already pointed out, the shutdown of Uddevalla unit was followed by the opening of Autonova some years later. But, in June 2001, the TT News Agency reported that Volvo Cars, subsidiary of Ford Motor Company, announced the decision of reintroducing the conventional assembly line in this plant. This decision from Volvo seemed to confirm the end of the "Volvo-ist" model. The executive management board and representatives from the Metallurgical Union agreed that this was the only way to ensure the future of the plant. On this matter, Volvo's Metallurgical Union President, Mattias Jonsson, declared that the abandon of "reflexive production" was a regrettable decision. Nevertheless, with the maintenance of jobs in stake, he also stated that it was not possible to argue in favor of such a production system. Basically, Jonsson's words synthesized the idea that the conventional assembly line was a matter of survival for the plant.

\section{TWO CASES IN THE AUTOMOBILE INDUSTRY}

This paper's empirical section is based on two case studies pointed to two companies from the automotive components industry. These companies - Huf and Iber-Oleff - have 391 and 320 (Dec. 2004) employees, respectively. The first company, located in the north of Portugal, belongs to an European group. The second one, located in the centre of Portugal, has resulted from a joint-venture between a Portuguese and a German group. Both companies combine different types of assembly lines (regarding shape, automation levels, etc.) and therefore they seem to be a privileged fieldwork considering the LiMITE project' focus. The interviews address different hierarchical levels, always including operators that work within different assembly lines systems.

Huf Portugal manufactures electronic and mechanic lock systems for the automobile industry. Amongst their products, for example, are the locks for the Opel Corsa and Astra, Ford Focus and Peugeot 307. Furthermore, it also manufactures blocking systems for the steering and external handles for doors. The company started operating on 1991. Iber-Oleff started production in 1992 in the area of plastic technical components, namely the air diffusion system for the Multi Purpose Vehicle produced by AutoEuropa. This company produces plastic functional components, ashtrays, air diffusers, speaker grills, ventilation grills, command systems for ventilation and air conditioning, parts and covers for car radios. 


\subsection{From the layout ...}

At Huf Portugal we can distinguish, even though if only roughly, three periods corresponding to three assembly line concepts. Naturally, within each of these periods co-exist assembly lines that are the answer to different concepts. In any case, at present, there are assembly lines in the factory layout which have very different formats and levels of technological sophistication.

In any case, during the launch period (phase 1), between 1991 and 1992, the lines were installed following mainly a traditional model - straight-line format, sequential logic, little technological sophistication and manual work. The assembly lines of the following period (phase 2), already with a vertical development, present a $U$ shape and started to include elevators (buffers) near each workplace - "They introduced the elevators and the boxes to accumulate material between workplaces and the material stopped accumulating, and thus breaking, between workplaces", explains one of the operators in the PSA (Peugeot-Citroen) line. In 2002 (phase 3), a new concept started to be planned. But even nowadays, the phase 2 system is kept for some products such as some of GM's (General Motors) products (steering blocking). There are also hybrid lines, such as the Ford line, where they produce sequentially and without large intermediary warehouses (the warehouses are the spaces between the equipment). The most advanced system is that of the new PSA line, that already incorporates more functions and also has the peculiarity that it combines the two products typical of Huf Portugal in the same line - on the one hand the locks, on the other, the blocking systems.

The new PSA line, phase 3, emerged following a partnership between Huf Portugal and Huf Germany. The former does not have a commercial department, meaning it is the Germans who agree the contracts with the clients. A new market opportunity arose and the possibility to increase the PSA market quota with this contract, from $20 \%$ to $60 \%$, hence the investment to create this new infrastructure. As the production manager noted, "It is important to be competitive amongst the different Huf companies. We design our layouts to absorb a larger chunk of the market, that is so that Huf Germany gives us more market. We never say no." In a hypothetical situation, he adds, "Let us suppose that a line was designed to produce 1400 sets. But if the client asks us for 3200 we have to set up another line, another structure. We have to invest."

These new lines, in installation and testing phase, are characterised by their serpentine format, high level of automation and the fact that in the same infrastructure, not one, but several different products are manufactured. In this model, the intermediary levels (buffers) are again suppressed. Which does not mean that between two equipments, due to some problem that makes a workplace slower than the others, there may not in effect exist a small number of stocks - it generally does not happen, but, when it does, that situation is due, mostly, to some problem in the equipment or to a problem in the quality of some component. In any case, the process is now supported by pallets (and no longer in boxes). In the lines introduced in phase 2, as we mentioned, the sets move inside boxes that, in turn, are accumulated in elevators. In this new model, "There are no boxes, there are pallets, and it all follows in sequence.", summarises the line head of the new PSA. It is a very recent system, which means that, in the words of the same speaker, "The machines are not even in the right places yet. It is the experimental phase. We 
started last week. But we are improving. Today, I am producing more and tomorrow it will be better."

One of the advantages of these new lines is their flexibility, allowing the assembly of different types of products at the same time. In the previous PSA line, in a $U$ shape, it was possible to assemble different products, but it was a different situation. According to the production manager, "Product A went in and product A came out. In this line [new PSA] it is possible to introduce product $A$ and, immediately afterwards products $\mathrm{B}$ and $\mathrm{C}$. Then you branch out depending on the needs, such and such an operation. We have a common trunk [shared components] for the composition of the locks. The ignition is common to all the projects in the PSA line. Then the keys change, with the logotypes for Fiat, Citroen, Peugeot. (...) Previously, when the line was U shaped, we changed the machine. Even though they were machines that engaged quickly, there was always a certain loss of time."

In fact, perfectly sequential systems are more demanding from the point of view of equipment maintenance and availability of the equipment itself. By resorting to the concept of intermediary buffers, present in the lines introduced in phase 2 , small stops and breakdowns can be solved in 5 or 10 minutes with no effect on the line output. These buffers are present within the framework of the philosophy of "n-1" people with relation to the number of workplaces. Therefore, there is always a workplace that does not have the human resources to man it, which means that people must be rotated. In the systems introduced in phase 3 , any breakdown causes a stop in production, which also means that the support processes, such as maintenance, have to be rethought, so that everything works efficiently and within the predicted rhythm. With reference to that, as opposed to the U-shaped lines that incorporate a support for components lasting about two hours (meaning that if the equipment stops, production carries on), in totally sequential lines, tolerance is limited to a few boxes, so that, if there is a breakdown, it is more probable that the production flow has to be interrupted.

At Iber-Oleff the VW240 (Volkswagen) assembly line (ventilation grills, command systems for ventilation and air conditioning) was installed about 5 years ago. The layout was conceived by the German company, an expert in line manufacture and assembly. There were several studies around the assembly line, until they reached the configuration that is essentially maintained to date. "Because I don't know if you noticed, we have a conveyor from behind that picks up the parts for painting down here and surely was taken into account for the layout." The industrial engineer also adds "We agreed with and criticised their work in a positive way. We will question as to why they are here and not there. Because we think that if it were somewhere else we would have these and these advantages. We face each other this way." The VW 240 assembly line has separate lines where the parts circulate, in different stages of product insertion. As a consequence, the same part is placed on different lines depending on the number of products that have been incorporated into it. The conveyor belt rotates continuously and depending on the task, the operator gets the product from the corresponding line.

From the time the line was installed, some suggestions have been made for alterations, but which did not compromise the structure of the initial project. "This type of alterations takes place a lot here. We constantly work on it. We change the layout very often.", mentions the production manager. In the same line, says the area head that "[the layouts are altered] within a matter of months." The proposals 
usually arise when the line is already working, as it is then that some alterations seem advantageous so that it is optimised. Concluding that in the case of the automobile industry the lines are usually connected to a product

There are adjustments that have been made a little at a time and that, from the point of view of the engineer that at the time oversaw the installation, should be reported to the Germans so that the information can be used, and when new lines are created they can incorporate that knowledge. It is a capital that is worth accumulating, re-using it in the assembly lines. Speaking of this, this engineer explains that "We can make those alterations, sometimes we report them, other times not, depending on how important we decide they are. If it is very important, and to collaborate with our experience, we report it. We evaluate everything, from ergonomics, the chair itself, and the height of the table. All aspects, may be one or so may escape us at first sight, but we always pay attention so that we don't forget anything."

The proposals that come from the Portuguese factory, in general, do not face great obstacles from the company responsible for the layouts. On this subject, the industrial engineer recounts another experience: "Just today I had a meeting to define a new assembly line, where it had been predicted that a certain piece of equipment should be in a certain place and here, we thought not, and suggested a change and they are going to accept the change because it is the most appropriate for that specific piece of equipment."

The discourses agree with regards to the advantages of the layout of the VW240 line: "We have advantages in terms of flexibility and balancing. The advantage is the flexibility and a better balancing, i.e. I can see if the work phases (process) are balanced or not, and they give the operator flexibility in his/her work. In terms of productivity, we do not impose but let the operator do the managing. The result is obvious, no need to call attention to it", says the production manager.

When we asked to the industrial engineer about the disadvantages of this line, the answer is conclusive: "There are no disadvantages." As for the extension of this configuration in the industry, the importance of the product is stressed. There are products for which "We cannot even think of that layout model. The basis for this part is this back part. On top of this back part which we call the housing, the lamellae are mounted, as well as a valve, a sponge, an axis, we assemble a link and a wheel (...). We assemble all this. It makes sense, I have the part on the first line and as I add operations I change the part to other lines until we reach the end where the last operator fits in the last part and then places it in the box." This type of configuration is shown as advantageous when there is a basic product and operations are added to the same part.

\section{2. ... to the work organisation}

It is the time to go into the question that is at the origin of the project LiMITE. In other words, to evaluate the conditioning role technology plays on the organisational work model. On that subject, the engineering manager tells us the following: "There is always the possibility of organizing the work differently. Usually, the person who designs the assembly line thinks one way, and this is the way we use when work starts. Everybody assumes that it is the best way, but that is not always true. With the experience that the line heads have, their technical knowledge, which is far superior to ours, and that of other people, there are always a few adjustments to be 
made. The alterations are discussed, and are discussed amongst everybody." This optimisation of the layout, if it takes place, is the responsibility of the engineering and the production managers. Usually, it is always the person responsible for production who participates in the project development phase. When launching the project, it is also the production (in this case, the manager and line heads) that suggests alterations to be discussed with the engineering manager. Picking up the initial question again, how does the technical aspect rule the other aspects? It varies. But, for example, it has been concluded that in the new PSA line, the way the equipment was conceived does not make it possible to advance to another workplace, to have access to the components.

In any case, and since the technical and organisational subsystems do not coexist independently from each other, together with the advances in the assembly infrastructure, the management philosophy has also evolved somewhat. Quoting the human resources manager, "The first one was of the type Chaplin's Modern Times, where each operator worked at a single post [one person for a workplace], there was no movement, no rotation. Therefore, a traditional assembly line. Then, we introduced working groups, continuously improving, and started to implement a more modern and more flexible assembly line philosophy." Following this reasoning, she explains, "The rhythm of the assembly line is set by the first workplace. What cannot be recovered in one workplace can be recovered in another. Automatically, our flexibility started in the middle stations of the assembly lines." However, in the opinion of the engineering manager, the introduction (on its own) of the $U$ line does not alter the working model significantly. "Working in a $U$ or in a line, for me, is the same concept. The only reason for working in $U$ is that there isn't enough space to place twelve pieces of equipment. The line was just bended to gain space."

In the new assembly lines, still in the development phase, but where in reality work is already being carried out, the alterations introduced in terms of work organisation are not substantial. In the words of an operator in the new PSA line, it turns out to be "Better, because we work facing the machine, we do not need to move so much. I think it is more cost-effective, small parts, we work with pallets, each one takes a set, all one after the other, always moving, with no elevators. [Do you then prefer to work without elevators?] That way the machine is in front of us, all we have to do is take it from the machine and place it on the pallet, whereas on the other line [with elevators], we used much bigger boxes and had to work with them on the machine - remove, turn, place - which meant more work." According to the engineering manager, in the transition from phase 2 to phase 3, "What happens is that the workplace as we understood it in concept 2 is more restricted in concept 3." That is, the tasks are more dispersed, which indicates some automation of the work.

But, still according to the engineering manager, "The organisation itself, the day to day of a person who works with this system, will not vary a lot. There is some specificity of one or other pieces of equipment, but not of the way people work." This idea is confirmed by an operator in the new PSA line: "The work is the same. It's just that in the old line everything was very manual." Very similar, the testimony of a colleague: "It is different because there are several robots (with regards to that we have evolved a bit) which now mount several things, may be it is not so hard, but, essentially, it is the same thing." That is, if it is true that the investment on electronic equipment in phase 3 brought in more flexibility, better technical 
knowledge and better sensibility, in what is essential, the work model ends up by not being that different from the previous situation. In terms of tiredness, the new technical infrastructure will not bring many changes, according to the declaration of the engineering manager himself: "It does not lighten the work load. We still take into account the same technical factors of tiredness in the time measuring studies."

At Iber-Oleff, the configuration of the line conditions a great deal the organisation choices. The structural options take place at the time that the layout is defined, there are options to choose from. Even though the content of the workplace remains, in general, very poor, there is a group logic that this type of configuration facilitates. Sharing the conveyors from which the operators remove the circulating parts, even though in a sequential logic, creates a team dynamic that usually escapes the work in a simple sequential line. "When they are in a sequential line, people are near each other and anything could happen. Whereas, there, we are sharing. There is an opening to the work team." The rhythm imposed by the conveyor belts and the limited content of the operations brings us near the traditional concept that we usually associate to the work in the assembly line. "The line itself helps people to gain the appropriate rhythm, that is, it almost creates a common rhythm.", says the production manager. However, there is a different dynamic imported from the variation of the sequential configuration of the line, which seems to restrict the limitations due to different rhythms and diminish the isolation of the workplaces and the dependence on each other.

It is worth stressing the tasks that are difficult to substitute by technical equipment, at least in an affordable way. Automation is emphasised, there is no doubt that the company is moving in that direction. Maintenance, or not, of the workplaces depends greatly on the technical advantages of the equipment and the return on the necessary investment for its acquisition. There are advantages in human work that seem to favour certain workplaces, just as there are technological investment costs that in some cases do not seem to compensate.

Automation usually means fewer jobs, even though those tasks are very repetitive. The area head tells us that "They have fewer workplaces. But, in any case, they are usually repetitive tasks. And sooner or later they would bring trouble. If we did not rotate people, surely tendonitis and such would emerge..."

The VW240 line has not suffered significant alterations in terms of the technologies used. The adjustments made to the line are connected, mainly, to the displacement of some workplaces, or the introduction of others. It is worth mentioning that production quantities take more people to that line. That is, when production increases, some workplaces are activated, similar to others that already exist in the line, in order to increase the resources available and that way to guarantee a response to production demands. But this organization counts on the subassys (lamellae assembly) operators who in these cases are allocated to the VW240 line. In other cases, they move operators from other areas to work in this line. There is a combination of efforts to fulfil the objectives established.

\section{FINAL REMARKS}

In relation to the question that encompasses the whole of the LiMITE project within the framework of this technology, is there margin for new organisational choices? - and to which we have been answering throughout the case studies, the 
Huf's human resources manager answered in these terms: "Always, processes, ways of doing things, can be improved."

On the one hand, the different assembly lines are the basis of the technical infrastructure of these factories, which means that, objectively, they define restrictions in terms of execution times and gestures for the operators, which is, to start with, a limitation. However, the two companies have been able to find relatively innovative ways to qualitatively optimise their work practices - and, amongst the different strategies they have initiated, it is worth mentioning the task rotation system (well organised) and management (in the hands of the operators themselves). Underneath this structure it was possible, in spite of everything, to find some elements that seem to contradict some painful characteristics usually associated to this type of work. It is precisely this cross-over between technological restriction and qualitative innovation that make Huf Portugal and Iber-Oleff interesting case studies within the conceptual framework of the LiMITE project.

\section{ACKNOWLEDGMENTS}

This work has been supported by the LiMITE project. A project funded by the Operational Program for Employment, Training and Social Development, sponsored by the Portuguese Ministry for Labour and Social Solidarity.

\section{REFERENCES}

1. Bergreen, C., Alternatives to Lean Production: Work Organization in the Swedish Auto Industry, New York: ILR Press, Capitulo 5 - Organizational and Technical Design of Swedish Automotive Industry, 1992, pp. 90-100.

2. Blauner, R. Alienation \& Freedom - the Factory Worker and his Indwstry, Chicago: The University of Chicago Press, (1964).

3. Coriat, B. L'Alelier et le Chronomètre: Essai sur le Taylorisme, le Fordisme et la Production de Masse, Paris: Christian Bourgois Éditeur, Cap. 14 - La Chaine, 1994, pp. 67-85.

4. Durand, J-P.; Stewart, P.; Castillo, J-J, Teamwork in the Automobile Industry - Radical Change or Passing Fashion?, Macmillan, GERPISA, 1999.

5. Emery, F., The Assembly Line: Its Logic and Our Future, Occasional Papers in Continuing Education, No. 7, 1975.

6. Engström, T.; Jonsson, D.: Medbo, L., "The Uddevalla plant and interpretations of industrial design process", Inlegrated Manufactwing Systems, Vol, 9, 5, MCB University Press, 1998, pp. 279-295.

7. IMT1, Mamfacturing processes \& Equipment Roadmap, Section 5-Assembly, 24 July 2000.

8. Jürgens, U., Rolling Back Cycle Times: the Renaissance of the Classic Assembly Line in Final Assembly, in: Shimokawa, K.; Jürgens, U.; Fujimoto, T. (eds), Transforming the Automobile Assembly: Experience in Automation and Work Organization, Berlin: Springer, 1997, pp. $255-273$.

9. Kahaner D K, Assembly Line Automation Activities in Japan, Asian Technology Information Program (ATIP), Japan, 1993.

10. Lopes, J. M., O Problema do Layout: tma Visão Global, dissertação de doutoramento, Braga: Universidade do Minho, 1990.

11. Rekiek, et al., "State of the art of optmization for assembly line design", Amntral Reviews in Control, 262002 , pp. 163-174.

12. Shimokawa, K.; Jürgens, U.; Fujimoto, T., Transforming the Altomobile Assembly: Experience in Automation and Work Organization. Berlin: Springer, Introdução, 1997, pp. $1-16$.

13. The Sky Group, The Product Layout - a facility layout concept, Production/Operations Management, on-line.

14. Trist ef al., Organizational Choice, London: Tavistock Institute, 1963

15. Walker, C. R.; Guest, R. H., The Man on the Assembly Line, Massachussets: Harvard University Press, 1952.

16. Wild, R., "On the selection of mass production systems", International Journal of Production Research, Vol. 13, No. 5, 1975, pp. 463-61. 